\title{
Biochemistry and physiological functions of ADAMTS7 metalloprotease
}

\author{
Hayley A. Hanby, X. Long Zheng* \\ Department of Pathology and Laboratory Medicine, The Children's Hospital of Philadelphia and The University of Pennsylvania Perelman \\ School of Medicine, Philadelphia, PA 19104
}

\section{Email address:}

zheng@email.chop.edu(X. Long Zheng)

\section{To cite this article:}

Hayley A. Hanby, X. Long Zheng. Biochemistry and Physiological Functions of ADAMTS7 Metalloprotease. Advances in Biochemistry. Vol. 1, No. 3, 2013, pp. 43-50. doi: 10.11648/j.ab.20130103.11

\begin{abstract}
Here, we provide a comprehensive review of current findings concerning the biochemistry and physiological functions of ADAMTS7, a metalloprotease that is known to interact with cartilage oligomeric matrix protein, progranulin, and alpha2-macroglobulin. Such broad substrate specificity and potentially diverse physiological functions make ADAMTS7 an interesting enzyme to study. ADAMTS7 has been shown to play a role in the pathogenesis of arthritis and disc disorders. More recently, the ADAMTS7 locus is identified to have a strong association with coronary atherosclerotic disease. However, the role of ADAMTS7 in the development of atherosclerosis is yet to be determined. The development of an easy and high throughput assay for ADAMTS7 activity and appropriate animal models will allow us to uncover the novel mechanisms of coronary arterial disease.
\end{abstract}

Keywords: ADAMTS7, Substrate Specificity, Structure-Function, Cardiovascular Disease, and Smooth Muscle Cell

\section{Introduction}

ADAMTS7, a member of A Disintegrin And Metalloprotease with ThromboSpondin type 1 repeats (ADAMTS) family, was first identified and cloned in 1999 [1]. The family of proteases consist of 19 secreted enzymes with proteolytic activity against extracellular substrates, especially extracellular matrix proteins $[2,3]$. They share a similar domain organization comprising a signal peptide, a prodomain, a metalloprotease domain, and various lengths of C-terminal domains including one to several thrombospodin type 1 repeats (TSP1), interspaced by Cys-rich and/or spacer domains, as exemplified by ADAMTS7 (Figure1). The importance of ADAMTS proteases is implicated in the establishment of tissue architecture during development and in tissue degradation during disease states such as cancer, Alzheimer's disease, and chronic inflammatory conditions $[2,3]$. Three specific functions have emerged through the characterization of several ADAMTS members, and they include the processing of procollagen by ADAMTS2 [4, 5], the degradation of chondroitin sulfate proteoglycans by ADAMTS4 [6-8] or ADAMTS5 [9-12], and the cleavage of von Willebrand factor (VWF) by ADAMTS13 [13-15].

Recently, two independent genome-wide association studies (GWAS) have identified the ADAMTS7 gene as a novel locus for the development of coronary atherosclerosis [16, 17]. Individuals carrying a single nucleotide polymorphism (SNP) in the ADAMTS7 gene (i.e. rs1994016) had a 19\% increase in the risk of developing atherosclerotic disease. Interestingly, the same study found that ADAMTS7 was not associated with myocardial

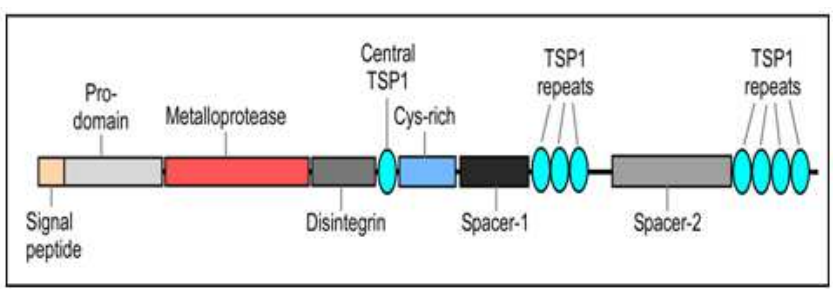

Figure1. Schematic structure and domain organization of ADAMTS7. Human ADAMTS7 consists of a conserved signal peptide, prodomain, and metalloprotease domain. The C-terminus of ADAMTS7 contains a disintegrin domain, the first thrombospondin type 1 repeat (TSP1), Cys-rich domain, and 7 additional TSP1 repeats interspaced with two spacer domains. The second spacer domain is also a mucin-like domain.

infarction (MI), suggesting that ADAMTS7 participates in the development and early progression of atherosclerosis but not thrombosis leading to MI.

To date, only three potential substrates for ADAMTS7 have been reported through yeast-two hybrid screens. 
These include cartilage oligomeric matrix protein (COMP) [18], progranulin (PGRN) [19], and alpha2-macroglobulin $(\alpha 2-\mathrm{MG})$ [20]. Nonetheless, little is known about the structure-function relationship and regulation of ADAMTS7 under pathophysiological conditions. This review focuses on the recent progress in the field concerning the biochemistry and physiological functions of ADAMTS7, relevant to the understanding of pathology and the development of novel prevention and therapeutics of arthritis and cardiovascular diseases.

\section{Expression and Localization}

The ADAMTS7 gene was found to be expressed in mouse embryos as a single transcript of $\sim 5.5 \mathrm{~kb}$, whereas in adult human samples, ADAMTS7 expression was detected (also $5.5 \mathrm{~kb}$ transcript) in the heart, pancreas, kidneys, skeletal muscle, and liver [21] In skeletal muscle, two additional transcripts at $8.0 \mathrm{~kb}$ and $4.5 \mathrm{~kb}$ were found, corresponding to alternative splice forms. In a later study, RT-PCR in various rat tissues detected ADAMTS7 mRNA in the liver, embryo, ovaries, kidneys, testicle, lung, and thymus, and at lower levels in the spleen, heart, and brain [18]. Similarly, ADAMTS7 mRNA is found in normal human bone, cartilage, synovia, tendon, and ligament as well as in meniscus, skeletal muscle, and fat at lower levels. As of now, the expression pattern of the ADAMTS7 transcript and protein in adult mice has not been characterized.

Overall, ADAMTS7 exhibits a broad tissue distribution but at low levels. The physiological implications of this widespread tissue expression of the ADAMTS7 gene remain to be determined.

Depending on the cell type, recombinant ADAMTS7 seems to be associated with the cell membrane or extracellular matrix. Conditioned medium of HEK293 cells stably expressing Myc-tagged murine ADAMTS7 exhibited a molecular weight of $250 \mathrm{kD}$ and larger by SDS-polyacrylamide gel electrophoresis (PAGE) and Western blot. ADAMTS7 appears to be heavily glycosylated, therefore, the molecular weight of the secreted ADAMTS7 from cells is larger than that determined by protein sequence analysis. Treatment of stable cells expressing ADAMTS7 with $0.5 \mathrm{M} \mathrm{NaCl}$ for $30 \mathrm{~min}$, which released more ADAMTS7 in the conditioned medium, supports the theory of cell surface and/or matrix localization of secreted ADAMTS7. Additionally, ADAMTS7 was detectable by surface biotinylation experiments, and in some cells, recombinant ADAMTS7 appears to be transiently associated with the outer leaflet of the plasma membrane. Depending on the concentration and composition of the extracellular matrix, a substantial fraction of total secreted ADAMTS7 protein may be cell-bound, although the structural feature governing cell or matrix association is not fully understood. In the context of disease, ADAMTS7 is localized to vascular smooth muscle cells (VSMCs) in human coronary and carotid atherosclerotic plaques [22].

\section{Regulation}

All stages of atherosclerosis are regulated by pro- and anti-inflammatory cytokines. Similarly, vascular injury elicits various stimuli that regulate the expression of ADAMTS7 mRNA and protein, specifically in vascular smooth muscle cells. Both the mRNA and protein of ADAMTS7 were induced by the proinflammatory cytokines, tumor necrosis factor- $\alpha$ (TNF- $\alpha$ ) and interleukin-1 $\beta$ (IL-1 $\beta$ ), and by growth factor platelet derived growth factor (PDGF)-BB (Figure2) [23, 24]. Reactive oxygen species

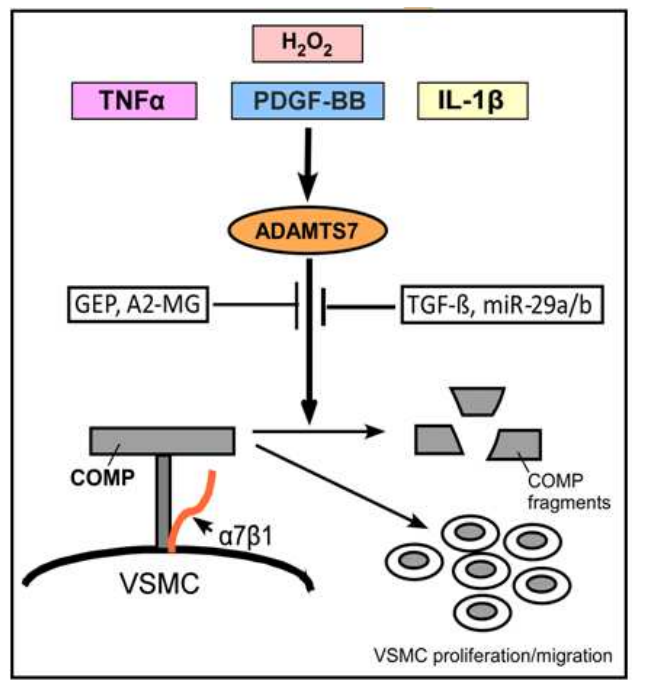

Figure2. Regulation of ADAMTS7 expression and its potential role in VSMCs. COMP is associated with vascular smooth muscle cells via binding to the $\alpha 7 \beta 1$ integrin and is cleaved by ADAMTS7. This proteolytic cleavage is regulated by several factors: inflammatory cytokines such as TNFa, $I L-1 \beta, T G F-\beta$; growth factor $P D G F-B B$; the reactive oxygen species $\mathrm{H} 2 \mathrm{O} 2$; microRNA miR-29a/b; and progranulin (PGN), and $\alpha 2-M G$. Cleavage of COMP has the highlighted physiological consequences such as cell proliferation and migration.

(ROS), such as hydrogen peroxide $\left(\mathrm{H}_{2} \mathrm{O}_{2}\right)$, also increased human ADAMTS7 expression. In contrast, the anti-inflammatory cytokine transforming growth factor- $\beta$ (TGF- $\beta$ ) downregulated ADAMTS7 expression (Figure2). Interestingly, proatherosclerotic factors, including oxidized low-density lipoprotein (oxLDL) and homocysteine, do not appear to alter ADAMTS7 expression.

Further studies have demonstrated that transcription factors, such as NF- $\kappa \mathrm{B}$ and $\mathrm{AP}-1$, which are essential for VSMC proliferation and migration, are also crucial for the regulation of ADAMTS7 expression in VSMCs [23]. Analysis of the ADAMTS7 promoter revealed the presence of proinflammatory element binding sites, including NF- $\kappa \mathrm{B}$ and AP-1 sites. TNF- $\alpha$ was shown to induce ADAMTS7 expression in human monocyte/macrophage cell line (THP-1), and a chromatin immunoprecipitation (ChIP) assay analysis confirmed the binding of NF- $\kappa \mathrm{B}$ and AP-1 to the corresponding sites within the ADAMTS7 promoter. These data suggest that ADAMTS7 expression is regulated at transcriptional levels in response to inflammatory stimuli under pathophysiological conditions. 
Recently, a microRNA, miR-29a/b, was found to play a role in regulating ADAMTS7 expression (Figure2) [25]. MicroRNAs are short, 22-nucleotide, non-coding RNAs that function in mRNA degradation and suppression of protein translation. Bioinformatics databases have predicted that the 3' untranslated region (UTR) of ADAMTS7 contains a target site for miR-29a/b. Co-transfection of VSMCs with double-stranded miR-29a/b mimic resulted in reduced activity of a luciferase reporter containing the 3 ' UTR of ADAMTS7. The miR-29a/b mimic also repressed ADAMTS7 mRNA and protein expression in VSMCs, whereas a single-stranded anti-miR-29a/b oligo, which inhibits endogenous miR-29a/b, increased ADAMTS7 expression levels. These data suggest that $\mathrm{miR}-29 \mathrm{a} / \mathrm{b}$ negatively regulates ADAMTS7 mRNA by targeting its 3 , UTR. Additionally, miR-29a/b is downregulated by NF- $\mathrm{kB}$, which has been shown to upregulate ADAMTS7 mRNA. However, the exact mechanism of how miR-29a/b regulates ADAMTS7 expression during inflammation remains to be elucidated.

A recent study on the coronary-artery-disease-association found a single nucleotide polymorphism (SNP; rs3825807) in patients with coronary artery disease, correlated with increased risk for the disease [22]. The SNP (A to G) renders a serine to proline substitution in the prodomain of ADAMTS7, which results in a reduction of ADAMTS7 expression and function in an in vitro study [22]. Other lead CAD-associated SNPs (rs1994016 and rs4380028) at the ADAMTS7 locus have yet to be investigated.

\section{Potential Substrates}

To date, three potential substrates have been identified to interact with ADAMTS7: COMP, PGRN, and a2-MG. COMP, also known as thrombospondin-5 (TSP5), a 520-kDa pentameric glycoprotein (Figure3) highly expressed in the cartilage of joints, vertebral discs, and vessels, was the first potential substrate of ADAMTS7, discovered by a yeast-2 hybrid screen for COMP binding partners. As bait, a construct of the EGF domains of COMP bound the C-terminal portion of ADAMTS7 [18]. The interaction between COMP and ADAMTS7 was further characterized by immunoprecipitation and degradation assays $[18,20]$. Liu et al demonstrated by a GST-pulldown assay that the four C-terminal TSP repeats of ADAMTS7 interacts with the N-terminal EGF-like domains of COMP. Unlike many other ADAMTS family members, the spacer domain, which confers substrate specificity, was not required for COMP binding. Additionally, recombinant rat ADAMTS7 was co-immunoprecipitated with COMP in native articular cartilage and the osteoblastic cell line MG-63, suggesting the physiological relevance of this interaction. More importantly, these investigators demonstrated that recombinant ADAMTS7 in conditioned medium may cleave COMP in in vitro digestion assays under the conditions described [18, 20]. The proteolytic cleavage of COMP by ADAMTS7

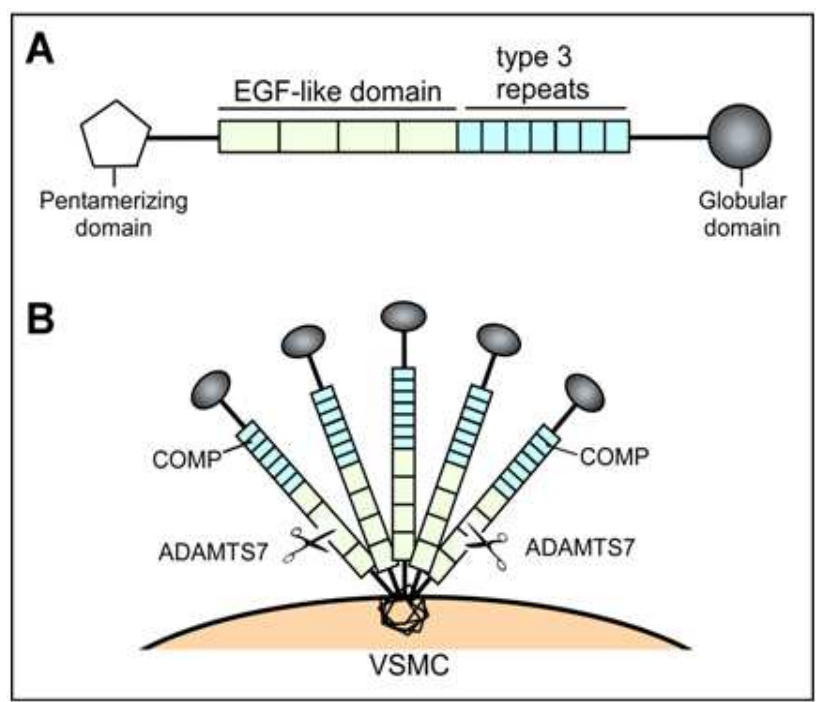

Figure3. Schematic structure and domain organization of COMP. A. Human COMP comprises a pentamerizing domain, four EGF-like domains, seven type 3 repeats, and a globular domain. B. A schematic diagram illustrates the pentameric form of COMP and potential proteolytic cleavage sites by ADAMTS7.

is calcium and zinc ion-dependent, as addition of EDTA abolished cleavage. COMP cleavage occurred at the physiological $\mathrm{pH}$ of 7.5, normal salt concentrations, and physiological temperature, yielding 3 potential fragments that migrated at

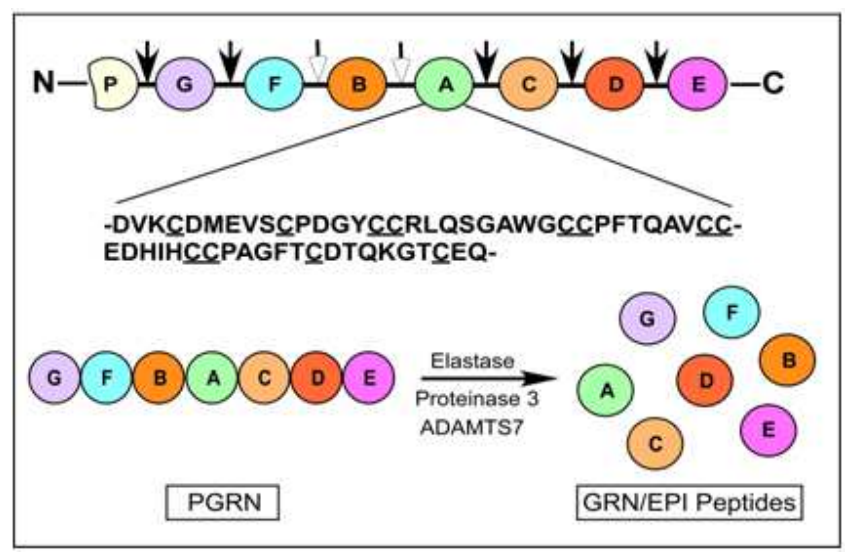

Figure4. Schematic domain organization and proteolytic processing of PGRN. Boxed letters indicate individual granulin domains. Scissors denote the elastase cleavage site and asterisks are the linker regions where proteolytic cleavage also takes place. However, the protease that releases granulins $A$ and $B$ has not been conclusively identified. The amino acid sequence of granulin $A$ is shown at the bottom as an example. Cysteines are underlined. Numbers below the sequence denote approximate positions of granulin domains in relation to full-length human progranulin.

100,75 , and $51 \mathrm{kD}$ on a SDS-PAGE under reducing conditions. Unfortunately, the exact cleavage sites on COMP by ADAMTS7 have not been identified.

PGRN, also known as plasma cell-derived growth factor, progranulin, acrogranin, or GP80 [27-29], binds ADAMTS7 and inhibits its proteolytic activity towards COMP [30]. PGRN was first discovered as a growth factor from conditioned medium, and proteolytic cleavage of PGRN 
liberates smaller units called granulins (Figure4), which possess biological activity of their own, such as inhibiting thrombin [31]. The precursor PGRN has also been implicated in development, mesothelial differentiation, and tissue repair [31, 32]. Prior studies demonstrated that the association between COMP and PGRN induces chondrocyte proliferation and that ADAMTS7 reduced PGRN-mediated chondrogenesis. Guo et al set out to examine this network of protein interactions, and they found that the four $\mathrm{C}$-terminal domains of ADAMTS7 were necessary and sufficient for association (like with COMP) with each and every PGRN subunit in the pericellular matrix of chondrocytes [19]. Although there is a claim that ADAMTS7 may be a novel PGRN convertase (Figure4), the experimental data are lacking to demonstrate the cleavage of PGRN by ADAMTS7 in vitro and in vivo. PGRN also regulates ADAMTS7 proteolytic activity and mRNA expression. PGRN was shown to disrupt the interaction between ADAMTS7 and COMP in a dose-dependent manner, and binding of ADAMTS7 to PGRN results in decreased COMP degradation by ADAMTS7 [19]. Additionally, PGRN inhibits TNF $\alpha$-induced ADAMTS7 expression in cultured human cartilage explants [19]. Interestingly, PGRN mRNA and protein expression levels are increased in arthritic cartilage compared to normal, healthy cartilage from the knee, suggesting the potential role of PGRN/ADAMTS7 interaction in the pathogenesis of arthritis. The elevated levels of PGRN, however, may not be sufficient for inhibition of ADAMTS7 and subsequent reduction of COMP degradation. Similarly, PGRN itself possesses inflammatory functions apart from its interaction with ADAMTS7 [33].

Alpha2-MG, a tetrameric plasma protein, is assembled from pairwise disulfide-bridged $180-\mathrm{kDa}$ subunits. This highly abundant plasma protein has broad specificity for trapping proteases. Binding of a protease to $\alpha 2-\mathrm{MG}$ results in "rapid" and "slow" conformational changes of $\alpha 2-\mathrm{MG}$. In addition, proteases cleave $\alpha 2-\mathrm{MG}$ at the bait region, resulting in an engulfment of the protease and formation of an irreversible complex (Figure5) [26]. In the case of ADAMTS7, binding to $\alpha 2-\mathrm{MG}$ generates a smear of high molecular weight complexes that migrate more slowly than the tetrameric or dimeric form of $\alpha 2-\mathrm{MG}$ by SDS-PAGE [20]. Additionally, $\alpha 2-\mathrm{MG}$ competitively inhibits the degradation of COMP by ADAMTS7 in vitro [20]. Inhibition of ADAMTS7 is a worthwhile avenue to explore, especially for the development of therapeutic agents against diseases, such as atherosclerosis and arthritis, in which ADAMTS7 has been implicated. But first and foremost, a better understanding of the enzyme itself must be achieved in order to begin to develop inhibitors against ADAMTS7. Data available to date indicate that $\alpha 2-\mathrm{MG}$ is unlikely to be a physiologically relevant substrate for ADAMTS7, but it is rather a sophisticated binding partner in circulation.

It is worth mentioning that ADAMTS7 was unable to cleave aggrecan and versican, common substrates of several ADAMTS family members [21]. This suggests that
ADAMTS7 is most likely not a redundant enzyme in the family, and it, like ADAMTS13, probably possesses one or several unique substrates.

\section{Structure-Function Relationship}

Hurskainen et al first identified ADAMTS7 with two thrombospondin repeats, having a similar domain structure to ADAMTS5, ADAMTS6, and ADAMTS8 [1]. This form was in fact an alternative splice variant arising from a consensus splice donor site in the intron [16]. A longer form of ADAMTS7, or ADAMTS7B, was later characterized as the full-length version of the enzyme [21]. The ADAMTSs are structurally related with the ADAM proteases and more distantly to matrix metalloproteases (MMPs), according to the MEROPS database [34]. The catalytic domain of all families contains a highly conserved zinc-binding sequence HEXXHXXGXXH, in which three histidine residues coordinate the catalytic zinc ion. The ADAM family act as membrane-bound ectodomain sheddases and activate cell surface molecules, such as growth factors and adhesion receptors [35, 36]. Unlike ADAM proteases, ADAMTS enzymes possess a central thrombospondin type 1-like repeat, which is thought to bind extracellular matrix (ECM) and sulfated glycosaminoglycans such as heparin and aggrecan 2, [37-40]. Other C-terminal ancillary domains, such as a cysteine-rich domain and spacer domain, aid in substrate recognition and efficient cleavage. Unlike other metalloproteases, each ADAMTS member demonstrates an unique substrate specificity due to the various exosites located in the $\mathrm{C}$-terminal regions of the enzymes.

Human and mouse ADAMTS7 proteins consist of 1686 and 1641 amino acids long with predicted masses of 181 and $178 \mathrm{kDa}$, respectively [21]. The difference in length and size is derived from the prodomain and mucin domain, both longer in the human sequence. Human and mouse ADAMTS7 have a high sequence homology (67\% identity and $74 \%$ similarity). Both human and mouse full-length protease contain 8 thrombospondin motifs, one central motif following the disintegrin-like domain, and 7 repeats arranged in two clusters, with TSR4 and TSR 5 separated by a second spacer domain (Figure1). This second spacer domain possesses the mucin domain, which is enriched in serines, threonines, and prolines for O-glycosylation. Chondroitinase $\mathrm{ABC}$ treatment of a Flag-tagged construct of the mucin domain of mouse ADAMTS7 shifted its mobility in a gel, verifying chondroitin sulfate chain attachment to the mucin domain [21]. Both human and mouse sequences also contain a number of $\mathrm{N}$-linked glycosylation sites: 10 in human and 9 in mouse. Treatment of mouse ADAMTS7 with PNGase F to remove N-linked sugars resulted in faster migration of ADAMTS7 by SDS-PAGE.

Additionally, there are three conserved prodomain processing sites (R58, R60, and R220) for proprotein convertases, such as furin, in both human and mouse; however, the human sequence has four additional furin sites (R92, R218, R229, and R232). The prodomain seems to be 


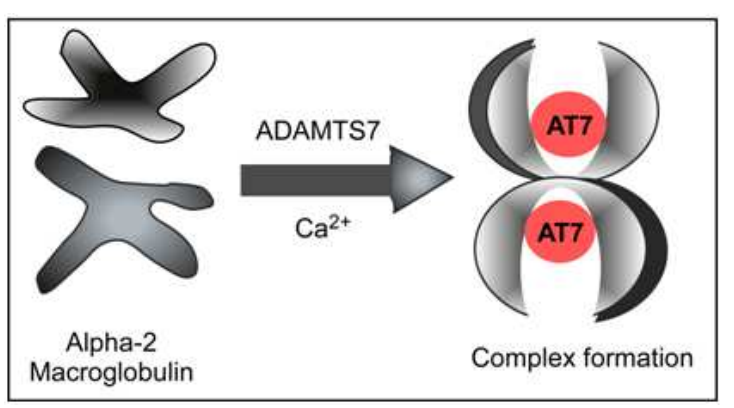

Figure5. Schematic representation of conformational change and protease trapping processes of $\alpha 2-M G$. Once a proteolytic enzyme such as ADAMTS7 (AT7) is absorbed to $\alpha 2-M G$, rapid conformational changes of the $\alpha 2-M G-A T 7$ complex occur. This process results in either an activation or an inhibition of the protease- $\alpha 2-M G$ complex, depending on the protease bound. In the case of AT7, $\alpha 2-M G$ trapping results in inactivation of AT7 proteolytic activity toward other substrates.

sequentially processed, as shown by a variant of mouse ADAMTS7 consisting of the prodomain and metalloprotease domain [21]. An initial 50-kD form was converted to a soluble $29-\mathrm{kD}$ metalloprotease form, with an intermediate $35-\mathrm{kD}$ form found at the cell surface. This result suggests that prodomain processing begins in the Golgi apparatus and is completed at the cell surface. Also, cell-type-dependent expression of furin may influence the prodomain processing and localization of ADAMTS7. Other proprotein convertases such as PACE4, PC6B, and PC7 were inefficient at full processing of the enzyme, suggesting that furin is the main proprotein convertase for ADAMTS7 [21]. This was also corroborated by the synthetic furin inhibitor decanoyl-RVKR chloromethylketone, which completely inhibited processing to smaller forms, rendering the zymogen the predominant form. This study also demonstrated that the single $\mathrm{N}$-glycosylation site in the prodomain aids in this processing. Since human ADAMTS7 contains four additional proprotein convertase sites, it would be interesting to determine the processing of its prodomain and whether other sites are preferentially cleaved instead of the conserved arginines.

Currently, several domains of ADAMTS7 have been implicated in binding to its substrates. However, the minimum domains required for efficient substrate cleavage is unknown. Both COMP and PGRN require four C-terminal TSP-1 repeats of ADAMTS7 for association, and expression of these domains alone is sufficient for binding to COMP and PGRN [19]. As for $\alpha 2 \mathrm{MG}$, the metalloprotease domain alone may be the only requirement for cleavage of this substrate. $\alpha 2-\mathrm{MG}$ contains a flexible bait region with high sequence variation, and the sites of cleavage within this bait region are dictated by the substrate specificity of the interacting enzyme [41]. Sequence diversity and localization of cleavage sites for proteinases were compared in five mammalian $\alpha 2-\mathrm{MG}$ proteins [41]. As mentioned previously, the spacer domains, especially the second spacer containing the mucin-like domain, do not seem to be required for binding to any ADAMTS7 substrate. Instead, the second spacer domain may be involved in localization of the enzyme to the cell surface or matrix. However, this hypothesis still needs to be investigated. The C-terminal ancillary domains of ADAMTS7 dictating substrate specificity remains to be identified, therefore a thorough structure-function study is necessary for a better understanding of this enzyme.

\section{Physiological Function}

For years, investigation of the physiological roles of ADAMTS7 has largely focused on its association with the pathogenesis of arthritis [18, 42] and disc diseases [43, 44]. This direction was undertaken by the observation that COMP is predominantly found in the extracellular matrix of cartilage. It was later shown that ADAMTS7 is also expressed in other COMP-producing tissues such as bone, cartilage, synovium, tendon, and ligament [18, 42]. ADAMTS7 is overexpressed in the cartilage and synovium of patients with rheumatoid arthritis [18, 42]. The COMP degradation fragments observed in the in vitro digestion assay with recombinant ADAMTS7 share the same molecular masses as those found in the synovial fluid in these patients with arthritic diseases [18].

Dynamic increases in ADAMTS7 expression and COMP degradation products were also observed in the nucleus pulpous during degeneration of rat caudal intervertebral disc [43]. Furthermore, Zhang et al also showed increased expression of ADAMTS7 mRNA and protein in degenerative intravertebral discs compared to non-degenerative discs [44]. ADAMTS7 acts as a negative regulator of endplate chondrocyte differentiation because it downregulates the expression of collagen type II, collagen type X, and Sox 9 [44], which are early and late marker genes for chondrogenesis. Therefore, under physiological conditions, the expression of normal levels of ADAMTS7 maintains the basal levels of COMP degradation in chondrocytes for chondrocyte differentiation. In pathological states where ADAMTS7 expression is upregulated, the excess COMP degradation results in degenerative and inflammatory diseases of the joints and intravertebral discs.

Recently, a role for ADAMTS7 in the pathogenesis of cardiovascular diseases has emerged. COMP is highly expressed in the normal VSMCs and has been shown to play an important role in maintaining vascular integrity, especially in the contractile phenotype of VSMCs through its interaction with the integrin $\alpha 7 \beta 1$ [45]. In a balloon injury rat model, ADAMTS7 accumulated preferentially in the neointima and mainly localized to the VSMCs. Overexpression of ADAMTS7 in VSMCs transduced by adenoviral vector encoding ADAMTS7 gene significantly accelerated cell migration and proliferation both in vitro and in vivo. Conversely, a siRNA-mediated knockdown of ADAMTS7 expression attenuated VSMC migration in culture through the degradation of COMP. Additionally, ADAMTS7 mediates the calcification of VSMCs through the degradation of COMP, which normally prevents calcification by inhibiting bone morphogenetic protein-2 
(BMP-2) osteogenic signaling [25, 46]. Cartilage oligomeric matrix protein inhibits vascular smooth muscle calcification by interacting with bone morphogenetic protein-2 [46]. Vascular mineralization, or the abnormal accumulation of calcium phosphate crystals in the vessel wall, is a symptom of several diseases, including chronic renal failure, atherosclerosis, and diabetes mellitus, and ADAMTS7's most recent role in VSMC calcification suggests even more widespread physiological effects of its proteolytic activity.

\section{Conclusion}

ADAMTS7 is an intriguing enzyme with potentially widespread roles in a variety of physiological and pathophysiological events. ADAMTS7 appears to bind and cleave COMP, PGRN, and $\alpha 2-\mathrm{MG}$. However, other yet-to-be-identified substrates and inhibitors may still exist. The evidence to date suggests a broad role for ADAMTS7 in matrix remodeling, either in cartilage, the vessel wall, or other extracellular matrices. In addition to its role in the pathogenesis of arthritis and degenerative vertebral disc disease, recent GWAS have identified the locus containing the ADAMTS7 gene as being associated with the development of coronary atherosclerotic disease in humans. Further investigation of the biological function of ADAMTS7 in cell culture systems and in appropriate animal models may shed new light on the pathophysiology of coronary arterial diseases.

\section{Acknowledgements}

The work was partially supported by grants from National Institute of Health (HL007971 to H.A.H. with Dr. Douglas B. Cines as the Principal Investigator) and HL-11518701A1 to X.L.Z.)

\section{Nomenclature}

ADAMTS, A disintegrin and metalloprotease with thrombospondin type 1 repeats; ADAM, a disintegrin and metalloprotease; COMP, cartilage oligomeric matrix protein; a2-MG, alpha 2-macroglobulin; PGRN, progranulin; ECM, extracellular matrix; MMP, matrix metalloproteinase; VSMC, vascular smooth muscle cells; SDS-PAGE, SDS-polyacrylamide gel electrophoresis; BMP, bone morphogenetic protein; GWAS, genome-wide association study; kDa, molecular weight of kilo Dalton, and TSP1, thrombosponding type 1 repeats.

\section{References}

[1] E. C. Arner, "Aggrecanase-mediated cartilage degradation," Curr. Opin. Pharmacol. 2002, vol. 2, pp. 322-29

[2] X. H. Bai, D. W. Wang, L. Kong, Y. Zhang, Y. Luan, T. Kobayashi, H. M. Kronenberg, X. P. Yu, and C. J. Liu, "ADAMTS-7, a direct target of PTHrP, adversely regulates endochondral bone growth by associating with and inactivating GEP growth factor," Mol. Cell Biol. 2009, vol. 29, pp. 4201-19

[3] A. Bateman and H. P. Bennett, "Granulins: the structure and function of an emerging family of growth factors," J. Endocrinol. 1998, vol. 158, pp. 145-51

[4] Coronary Artery Disease (C4D) Genetics Consortium., "A genome-wide association study in Europeans and South Asians identifies five new loci for coronary artery disease," Nat. Genet. 2011, vol. 43, pp. 339-44

[5] A. Didangelos, U. Mayr, C. Monaco, and M. Mayr, "Novel role of ADAMTS-5 protein in proteoglycan turnover and lipoprotein retention in atherosclerosis," J. Biol. Chem. 2012, vol. 287, pp. 19341-45

[6] Y. Du, C. Gao, Z. Liu, L. Wang, B. Liu, F. He, T. Zhang, Y. Wang, X. Wang, M. Xu, G. Z. Luo, Y. Zhu, Q. Xu, X. Wang, and W. Kong, "Upregulation of a disintegrin and metalloproteinase with thrombospondin motifs-7 by miR-29 repression mediates vascular smooth muscle calcification," Arterioscler. Thromb. Vasc. Biol. 2012, vol. 32, pp. 2580-2588

[7] Y. Du, Y. Wang, L. Wang, B. Liu, Q. Tian, C. J. Liu, T. Zhang, Q. Xu, Y. Zhu, O. Ake, Y. Qi, C. Tang, W. Kong, and X. Wang, "Cartilage oligomeric matrix protein inhibits vascular smooth muscle calcification by interacting with bone morphogenetic protein-2," Circ. Res. 2011, vol. 108, pp. 917-28

[8] R. J. Fernandes, S. Hirohata, J. M. Engle, A. Colige, D. H. Cohn, D. R. Eyre, and S. S. Apte, "Procollagen II amino propeptide processing by ADAMTS-3. Insights on dermatosparaxis," J Biol Chem 2001, vol. 276, pp. 31502-9

[9] G. Gao, A. Plaas, V. P. Thompson, S. Jin, F. Zuo, and J. D. Sandy, "ADAMTS4 (aggrecanase-1) activation on the cell surface involves C-terminal cleavage by glycosylphosphatidyl inositol-anchored membrane type 4-matrix metalloproteinase and binding of the activated proteinase to chondroitin sulfate and heparan sulfate on syndecan-1," J Biol Chem 2004, vol. 279, pp. 10042-51

[10] C. Gendron, M. Kashiwagi, N. H. Lim, J. J. Enghild, I. B. Thogersen, C. Hughes, B. Caterson, and H. Nagase, "Proteolytic activities of human ADAMTS-5: comparative studies with ADAMTS-4," J. Biol. Chem. 2007, vol. 282, pp. 18294-306

[11] F. Guo, Y. Lai, Q. Tian, E. A. Lin, L. Kong, and C. Liu, "Granulin-epithelin precursor binds directly to ADAMTS-7 and ADAMTS-12 and inhibits their degradation of cartilage oligomeric matrix protein," Arthritis Rheum. 2010, vol. 62, pp. 2023-36

[12] Z. He, C. H. Ong, J. Halper, and A. Bateman, "Progranulin is a mediator of the wound response," Nat. Med. 2003, vol. 9, pp. 225-29

[13] T. L. Hurskainen, S. Hirohata, M. F. Seldin, and S. S. Apte, "ADAM-TS5, ADAM-TS6, and ADAM-TS7, novel members of a new family of zinc metalloproteases. General features and genomic distribution of the ADAM-TS family," J. Biol. Chem. 1999, vol. 274, pp. 25555-63

[14] M. L. Iruela-Arispe, D. Carpizo, and A. Luque, "ADAMTS1: a matrix metalloprotease with angioinhibitory properties," Ann N Y Acad Sci 2003, vol. 995, pp. 183-90 
[15] G. C. Jones and G. P. Riley, "ADAMTS proteinases: a multi-domain, multi-functional family with roles in extracellular matrix turnover and arthritis," Arthritis Res. Ther. 2005, vol. 7, pp. 160-169

[16] M. B. Jones, M. Spooner, and E. C. Kohn, "The granulin-epithelin precursor: a putative new growth factor for ovarian cancer," Gynecol. Oncol. 2003, vol. 88, pp. S136-S139

[17] K. Kuno and K. Matsushima, "ADAMTS-1 protein anchors at the extracellular matrix through the thrombospondin type I motifs and its spacing region," J Biol Chem 1998, vol. 273, pp. $13912-7$

[18] G. G. Levy, W. C. Nichols, E. C. Lian, T. Foroud, J. N. McClintick, B. M. McGee, A. Y. Yang, D. R. Siemieniak, K. R. Stark, R. Gruppo, R. Sarode, S. B. Shurin, V. Chandrasekaran, S. P. Stabler, H. Sabio, E. E. Bouhassira, J. D. Upshaw, Jr., D. Ginsburg, and H. M. Tsai, "Mutations in a member of the ADAMTS gene family cause thrombotic thrombocytopenic purpura," Nature 2001, vol. 413, pp. 488-94

[19] S. W. Li, M. Arita, A. Fertala, Y. Bao, G. C. Kopen, T. K. Langsjo, M. M. Hyttinen, H. J. Helminen, and D. J. Prockop, "Transgenic mice with inactive alleles for procollagen N-proteinase (ADAMTS-2) develop fragile skin and male sterility," Biochem. J. 2001, vol. 355, pp. 271-78

[20] C. J. Liu, "The role of ADAMTS-7 and ADAMTS-12 in the pathogenesis of arthritis," Nat. Clin. Pract. Rheumatol. 2009, vol. 5 , pp. 38-45

[21] C. J. Liu, W. Kong, K. Ilalov, S. Yu, K. Xu, L. Prazak, M. Fajardo, B. Sehgal, and P. E. Di Cesare, "ADAMTS-7: a metalloproteinase that directly binds to and degrades cartilage oligomeric matrix protein," FASEB J. 2006, vol. 20, pp. $988-90$

[22] Y. Luan, L. Kong, D. R. Howell, K. Ilalov, M. Fajardo, X. H. Bai, P. E. Di Cesare, M. B. Goldring, S. B. Abramson, and C. J. Liu, "Inhibition of ADAMTS-7 and ADAMTS-12 degradation of cartilage oligomeric matrix protein by alpha-2-macroglobulin," Osteoarthritis. Cartilage. 2008, vol. 16 , pp. $1413-20$

[23] H. Nagase and M. Kashiwagi, "Aggrecanases and cartilage matrix degradation," Arthritis Res. Ther. 2003, vol. 5, pp. 94-103

[24] X. Pu, Q. Xiao, S. Kiechl, K. Chan, F. L. Ng, S. Gor, R. N. Poston, C. Fang, A. Patel, E. C. Senver, S. Shaw-Hawkins, J. Willeit, C. Liu, J. Zhu, A. T. Tucker, Q. Xu, M. J. Caulfield, and S. Ye, "ADAMTS7 cleavage and vascular smooth muscle cell migration is affected by a coronary-artery-disease-associated variant," Am. J. Hum. Genet. 2013, vol. 92, pp. 366-74

[25] N. D. Rawlings, A. J. Barrett, and A. Bateman, "MEROPS: the database of proteolytic enzymes, their substrates and inhibitors," Nucleic Acids Res. 2012, vol. 40, pp. D343-D350

[26] M. P. Reilly, M. Li, J. He, J. F. Ferguson, I. M. Stylianou, N. N. Mehta, M. S. Burnett, J. M. Devaney, C. W. Knouff, J. R. Thompson, B. D. Horne, A. F. Stewart, T. L. Assimes, P. S. Wild, H. Allayee, P. L. Nitschke, R. S. Patel, N. Martinelli, D. Girelli, A. A. Quyyumi, J. L. Anderson, J. Erdmann, A. S. Hall, H. Schunkert, T. Quertermous, S. Blankenberg, S. L. Hazen, R. Roberts, S. Kathiresan, N. J. Samani, S. E. Epstein, and D. J. Rader, "Identification of ADAMTS7 as a novel locus for coronary atherosclerosis and association of $\mathrm{ABO}$ with myocardial infarction in the presence of coronary atherosclerosis: two genome-wide association studies," Lancet 2011, vol. 377, pp. 383-92

[27] R. P. Somerville, J. M. Longpre, E. D. Apel, R. M. Lewis, L. W. Wang, J. R. Sanes, R. Leduc, and S. S. Apte, "ADAMTS7B, the full-length product of the ADAMTS7 gene, is a chondroitin sulfate proteoglycan containing a mucin domain," J. Biol. Chem. 2004, vol. 279, pp. 35159-75

[28] L. Sottrup-Jensen, "Alpha-macroglobulins: structure, shape, and mechanism of proteinase complex formation," J. Biol. Chem. 1989, vol. 264, pp. 11539-42

[29] L. Sottrup-Jensen, "Alpha-macroglobulins: structure, shape, and mechanism of proteinase complex formation," J. Biol. Chem. 1989, vol. 264, pp. 11539-42

[30] H. Stanton, J. Melrose, C. B. Little, and A. J. Fosang, "Proteoglycan degradation by the ADAMTS family of proteinases," Biochim. Biophys. Acta 2011, vol. 1812, pp. 1616-29

[31] X. Sun, M. Gulyas, and A. Hjerpe, "Mesothelial differentiation as reflected by differential gene expression," Am. J. Respir. Cell Mol. Biol. 2004, vol. 30, pp. 510-518

[32] B. L. Tang, "ADAMTS: a novel family of extracellular matrix proteases," Int J Biochem Cell Biol 2001, vol. 33, pp. $33-44$

[33] M. Tortorella, M. Pratta, R. Q. Liu, I. Abbaszade, H. Ross, T. Burn, and E. Arner, "The thrombospondin motif of aggrecanase-1 (ADAMTS-4) is critical for aggrecan substrate recognition and cleavage," J Biol Chem 2000, vol. 275 , pp. $25791-7$

[34] M. D. Tortorella, M. Pratta, R. Q. Liu, J. Austin, O. H. Ross, I. Abbaszade, T. Burn, and E. Arner, "Sites of aggrecan cleavage by recombinant human aggrecanase-1 (ADAMTS-4)," J Biol Chem 2000, vol. 275, pp. 18566-73

[35] S. L. Turner, M. E. Blair-Zajdel, and R. A. Bunning, "ADAMs and ADAMTSs in cancer," Br. J. Biomed. Sci. 2009 , vol. 66 , pp. $117-28$

[36] M. N. Vankemmelbeke, I. Holen, A. G. Wilson, M. Z. Ilic, C. J. Handley, G. S. Kelner, M. Clark, C. Liu, R. A. Maki, D. Burnett, and D. J. Buttle, "Expression and activity of ADAMTS-5 in synovium," Eur. J. Biochem. 2001, vol. 268, pp. $1259-68$

[37] L. Wang, X. Wang, and W. Kong, "ADAMTS-7, a novel proteolytic culprit in vascular remodeling," Sheng Li Xue. Bao. 2010, vol. 62, pp. 285-94

[38] L. Wang, J. Zheng, X. Bai, B. Liu, C. J. Liu, Q. Xu, Y. Zhu, N. Wang, W. Kong, and X. Wang, "ADAMTS-7 mediates vascular smooth muscle cell migration and neointima formation in balloon-injured rat arteries," Circ. Res. 2009, vol. 104 , pp. $688-98$

[39] L. Wang, J. Zheng, Y. Du, Y. Huang, J. Li, B. Liu, C. J. Liu, Y. Zhu, Y. Gao, Q. Xu, W. Kong, and X. Wang, "Cartilage oligomeric matrix protein maintains the contractile phenotype of vascular smooth muscle cells by interacting with alpha(7)beta(1) integrin," Circ. Res. 2010, vol. 106, pp. $514-25$

[40] J. M. White, "ADAMs: modulators of cell-cell and 
cell-matrix interactions," Curr Opin Cell Biol 2003, vol. 15, pp. 598-606

[41] H. Yu and Y. Zhu, "Expression of ADAMTS-7 and ADAMTS-12 in the nucleus pulposus during degeneration of rat caudal intervetebral disc," J. Vet. Med. Sci. 2012, vol. 74, pp. $9-15$

[42] T. Zanocco-Marani, A. Bateman, G. Romano, B. Valentinis, Z. $\mathrm{H}$. He, and R. Baserga, "Biological activities and signaling pathways of the granulin/epithelin precursor," Cancer Res. 1999, vol. 59, pp. 5331-40

[43] Q. Zhang, M. Huang, X. Wang, X. Xu, M. Ni, and Y. Wang, "Negative effects of ADAMTS-7 and ADAMTS-12 on endplate cartilage differentiation," J. Orthop. Res. 2012, vol. 30 , pp. 1238-43
[44] X. L. Zheng, D. Chung, T. Takayama, E. Majerus, J. Sadler, and K. Fujikawa, "Structure of von Willebrand factor-cleaving protease (ADAMTS13), a metalloprotease involved in thrombotic thrombocytopenic purpura," J Biol Chem 2001, vol. 276, pp. 41059-63

[45] X. L. Zheng and J. E. Sadler, "Pathogenesis of Thrombotic Microangiopathies," Annu. Rev. Path. Mech. Dis. 2008, vol. 3, pp. 249-77

[46] J. Zhu, C. Nathan, W. Jin, D. Sim, G. S. Ashcroft, S. M. Wahl, L. Lacomis, H. Erdjument-Bromage, P. Tempst, C. D. Wright, and A. Ding, "Conversion of proepithelin to epithelins: roles of SLPI and elastase in host defense and wound repair," Cell 2002, vol. 111, pp. 867-78 